\title{
Airway accidents in critical care unit: A 3-year retrospective study in a Public Teaching Hospital of Eastern India
}

\author{
Sugata Dasgupta, Shipti Shradha Singh, Arunima Chaudhuri', Dipasri Bhattacharya, \\ Sourav Das Choudhury ${ }^{2}$
}

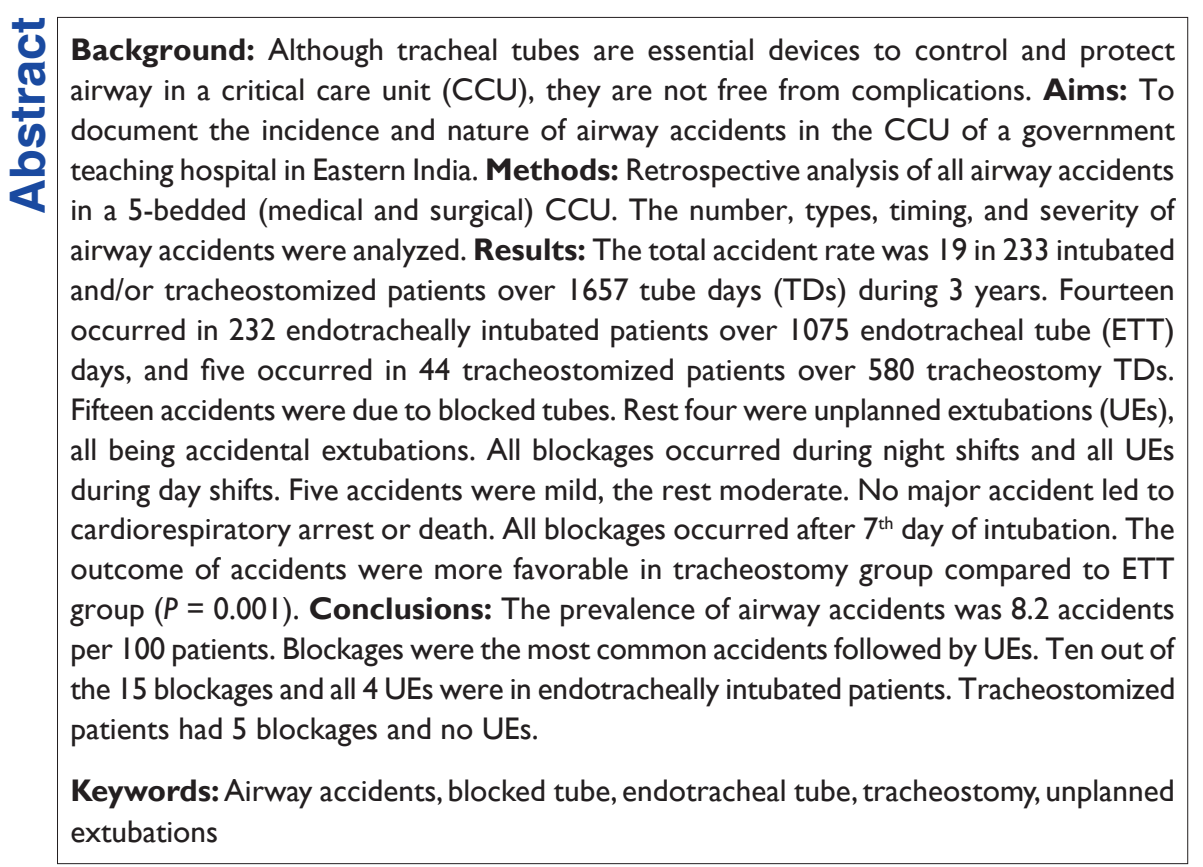

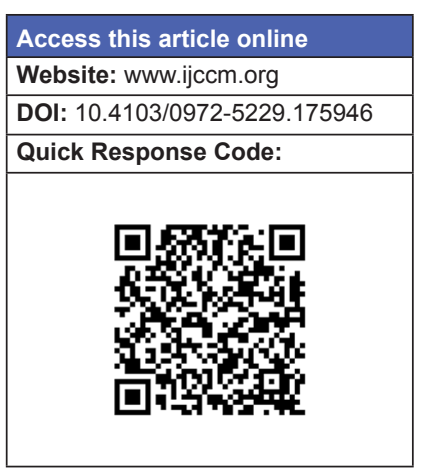

\section{Introduction}

Tracheal tube, either an endotracheal tube (ETT) or a tracheostomy tube (TT), is an essential device for controlling and protecting airway in the critical care unit (CCU). The use of tracheal tubes, however, is associated with complications such as unplanned extubation (UE) (both accidental and self-extubation [SE]), displacement, blockage (both ETT blockage and TT

\section{From:}

Department of Anesthesiology and Critical Care Medicine, RG Kar Medical College and Hospital, Kolkata, 'Department of Physiology, Burdwan Medical College and Hospital, Burdwan, ${ }^{2}$ Department of Critical Care, Behrampore, West Bengal, India

Correspondence:

Dr. Arunima Chaudhuri, Krishnasayar South,

Borehat, Burdwan - 713 102, West Bengal, India.

E-mail: arunimachaudhuri4u@gmail.com blockage), endobronchial intubation, kinking of tube, and leaking cuffs. ${ }^{[1]}$ The longer a tube stays in situ, greater is the chance of the airway accidents. ${ }^{[2]}$ These airway accidents can lead to life-threatening hypoventilation and hypoxia.

A few studies on UEs have shown an incidence ranging from $0.3 \%$ to $30 \% \cdot{ }^{[1-3]}$ Studies have also shown that a

This is an open access article distributed under the terms of the Creative Commons Attribution-NonCommercial-ShareAlike 3.0 License, which allows others to remix, tweak, and build upon the work non-commercially, as long as the author is credited and the new creations are licensed under the identical terms.

For reprints contact: reprints@medknow.com

How to cite this article: Dasgupta S, Singh SS, Chaudhuri A, Bhattacharya D, Choudhury SD. Airway accidents in critical care unit: A 3-year retrospective study in a Public Teaching Hospital of Eastern India. Indian J Crit Care Med 2016;20:91-6. 
significant proportion of patients having UEs tolerated it well, often not requiring reintubation. ${ }^{[4,5]}$ Most of these were associated with minimal or no morbidity. ${ }^{[6]}$ In the studies of late 1980s and early 1990s, SE has been reported to occur at the rate ranging between $8 \%$ and $16 \% \cdot{ }^{[4,7-12]}$ However, the rate dropped to around 3\% in studies of the late 1990s, attributed to active in-service education and ongoing clinical monitoring program. ${ }^{[13]}$

Indian studies on airway accidents in an Intensive Care Unit (ICU) or CCU are limited..$^{[1,2,14]}$ Exhaustive search in PubMed with keywords namely "airway accidents," "unplanned extubations," and "blocked tubes" revealed no published studies from Eastern India. To address this lacunae in literature, we carried out a retrospective study of all tracheal tube related accidents over 3 years (January 2012-December 2014) in a 5-bedded CCU (medical and surgical) under the Department of Anaesthesiology, Pain and Critical Care Medicine of a Government Teaching Hospital of Eastern India to evaluate the incidence, type, and medical impact of such accidents.

\section{Methods}

The records of all intubated and tracheostomized adult patients managed in the CCU between January 2012 and December 2014 were included.

\section{Protocols of airway care}

Polyvinyl chloride tubes with high volume-low pressure cuffs were used for endotracheal intubation. All ETTs were secured with an adhesive bandage applied to the lower chin and cheek along with a tie. All tracheostomies were inserted surgically into either a disc of trachea removed or a flap created. All TTs were secured with a tie around the neck. A tracheostomy was used after 10-20 days of endotracheal intubation or earlier if it was apparent that the patient was likely to require invasive ventilation for a long period. Heat and moisture exchanger-filters (HMEFs) were not available and heated humidifiers (HHs) were used for humidification.

All ventilated patients were kept adequately sedated with infusion of injection midazolam and fentanyl to maintain light sedation (Ramsay sedation scale 2-4 or Richmond agitation-sedation scale - 2-0). Sedation was stopped in the morning to assess neurological status or for spontaneous breathing trials as and when indicated. Weaning was done by recommended protocols. ${ }^{[15]}$ The conscious patients were not restrained but were repeatedly counseled not to touch the tubes. The drowsy, restless, and delirious patients had their hands gently tied down, which were released as soon as the sensorium improved. Pharmacologic (e.g., aerosolized inhalation therapies) and nonpharmacologic (e.g., chest physiotherapy) airway clearance therapies were applied as and when indicated.

\section{Staffing levels and supervision}

The nurse to patient (with an ETT/TT) ratio varied from 1:2.5 during the morning to 1:5 during evening and night. The respiratory technician, present during the day, assisted the on-duty nurses and doctors in the care of tracheal tubes. The CCU was exclusively staffed by the residents of the Department of Anaesthesiology, Pain and Critical Care Medicine. A senior anesthesiologist was always available in the hospital for help if needed. Airway protocols were strictly followed and constantly reinforced. All accidents were immediately reported to the chief nurse and consultant-in-charge of the CCU.

The tracheal tube accidents were categorized as follows

- UEs: SE if the patient intentionally removed the tube or accidental extubation (AE) if caused during movement for any reason

- Blocked tubes (blocked endotracheal tube [BET]; blocked TT [BTT]): Diagnosed if manual bag ventilation became diffi cult or mechanical ventilation showed high airway pressure or by noting that the lumen of the replaced tracheal tube was occluded requiring reintubation

- Displacement of tracheal tube: If the tip was seen displaced above vocal cords on laryngoscopy

- Endobronchial intubations: Diagnosed on auscultation when the air entry was not equal bilaterally

- Kinked tubes: Diagnosed by direct visualization under laryngoscopy

- Leaking cuffs: Diagnosed if there was large tidal volume leak compromising effective ventilation. ${ }^{[14]}$

\section{The severity of the airway accidents was graded as follows}

- Mild: Little or no physiological consequences; managed by resident doctor on duty

- Moderate: Cardiorespiratory decompensation; senior or experienced person required to manage the problem

- Major: Near or actual cardiorespiratory arrest or Death. ${ }^{[1]}$

All categorical data were expressed as proportions or percentages. Statistical analysis was performed using SPSS (version 20.0) (IBM Corporation, New York, USA). The categorical data analysis was done either by Fisher's 
Exact Test or Chi-square test as applicable. The numerical data were analyzed by unpaired $t$-test or ANOVA for normal distribution and by Mann-Whitney U-test or Kruskal-Wallis H-test if it was not distributed normally. The statistical significance implies $P<0.05$.

The Institutional Ethics Committee of the hospital approved the study.

\section{Results}

In the 3-year period for which the data were analyzed, 233 patients were intubated and/or tracheostomized for a total of 1657 tube days (TDs). Eight out of 136 female patients and 11 out of 97 male patients suffered airway accidents; the prevalence between the groups was comparable $(P=0.133)$. The mean and median age of patients were $41.18 \pm 20.745$ years (mean \pm standard deviation) and $36(23,59)$ (median [interquartile range]). Forty-four patients had tracheostomies, and all except one had an ETT placed before the surgical insertion of a tracheotomy tube. Therefore, a total of 232 patients had an ETT. TDs for patients with an ETT were 1077 and with a tracheostomy 580 .

The year wise distribution of airway accidents showed that number of airway accidents per TD was comparable between the years [Table 1]. No patient had more than one airway accident and 10 out of the 15 blockages and all 4 UEs were in ETT group. There was no case of displacement, endobronchial intubation, kinking of tube, or leaking cuff.

The incidence of accidents was similar between endotracheal intubation and tracheostomy $(7.18 \%$ vs. 13.51\%) $(P=0.198)$. However, the incidence was higher in the ETT group per TD compared to the tracheostomy group per TD $(1.3 \%$ vs. $0.9 \%)(P=0.001)$ [Table 1$]$. The incidence of accident was not affected by the departments from where the patients were referred $(P=0.483)$, but the type of accident had an association with the same $(P=0.036)$ [Table 1$]$. Three UEs occurred in obstetric patients, one in a medical patient, and none in surgical patients. Seven BETs were noted in medical patients, 3 in surgical patients, and none in obstetric patients. Three and two BTTs occurred in medical and obstetric patients, respectively; none occurred in surgical patients.

\section{Factors associated with occurrence and severity of airway accidents}

The severity (classified as mild, moderate, or major) of the accidents was higher in the ETT group compared to
Table I: Incidence of airway accidents; comparison of accident rates and severity by the type of tracheal tube; comparison of accident rates by referring departments

\begin{tabular}{|c|c|c|c|c|}
\hline $\begin{array}{l}\text { Yearly and total incidence of } \\
\text { airway accidents }\end{array}$ & $\begin{array}{c}2012 \\
(n=81)\end{array}$ & $\begin{array}{c}2013 \\
(n=58)\end{array}$ & $\begin{array}{l}2014 \\
(n=94)\end{array}$ & $\begin{array}{c}\text { Total } \\
(n=233)\end{array}$ \\
\hline Patients intubated/tracheostomized $(n)$ & 81 & 58 & 94 & 233 \\
\hline TDs $(n)$ & 605 & 464 & 588 & 1657 \\
\hline Airway accidents $(n)$ & 8 & 4 & 7 & 19 \\
\hline Airway accident/patient (\%) & 9.9 & 6.9 & 7.4 & 8.2 \\
\hline Airway accident/TDs (\%)* & 1.3 & 0.9 & 1.2 & I.I \\
\hline Accidental extubation $(n)$ & 2 & I & I & 4 \\
\hline Self-extubation $(n)$ & 0 & 0 & 0 & 0 \\
\hline $\operatorname{BET}(n)$ & 4 & 2 & 4 & 10 \\
\hline $\operatorname{BTT}(n)$ & 2 & I & 2 & 5 \\
\hline Displacement $(n)$ & 0 & 0 & 0 & 0 \\
\hline Endobronchial intubation $(n)$ & 0 & 0 & 0 & 0 \\
\hline Kinked tube $(n)$ & 0 & 0 & 0 & 0 \\
\hline Leaking cuff $(n)$ & 0 & 0 & 0 & 0 \\
\hline Total airway accidents $(n)$ & 8 & 4 & 7 & 19 \\
\hline \multicolumn{5}{|c|}{$\begin{array}{l}* P=0.992 \text { (Kruskal-Wallis H-test). TDs: Tube days; BET: Blocked endotracheal tube; } \\
\text { BTT: Blocked tracheostomy tube }\end{array}$} \\
\hline $\begin{array}{l}\text { Comparison of accident rates } \\
\text { by the type of tracheal tube }\end{array}$ & \multicolumn{2}{|c|}{$\begin{array}{l}\text { ETT group } \\
(n=232)\end{array}$} & \multicolumn{2}{|c|}{$\begin{array}{l}\text { Tracheostomy } \\
\text { group }(n=44)\end{array}$} \\
\hline Number of accidents & \multicolumn{2}{|c|}{14} & \multicolumn{2}{|r|}{5} \\
\hline Total number of patients & \multicolumn{2}{|c|}{232} & \multicolumn{2}{|r|}{44} \\
\hline Total number of TDs & \multicolumn{2}{|c|}{1077} & \multicolumn{2}{|r|}{580} \\
\hline Airway accidents/patients (\%) & \multicolumn{2}{|c|}{6.0} & \multicolumn{2}{|r|}{11.4} \\
\hline Airway accidents/TDs (\%)** & \multicolumn{2}{|c|}{1.3} & & 0.9 \\
\hline ** $P=0.00$ (Mann-Whitney U-test). TDs: Tub & be days; ET & TT: Endotr & acheal tube & \\
\hline $\begin{array}{l}\text { Severity of accident by } \\
\text { type of tracheal tube }\end{array}$ & $\begin{array}{l}\text { roup } \\
\text { \%) }\end{array}$ & $\begin{array}{l}\text { Trache } \\
\text { group }\end{array}$ & $\begin{array}{l}\text { ostomy } \\
n(\%)\end{array}$ & Total \\
\hline Mild & & 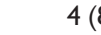 & 80) & 5 \\
\hline Moderate & .86) & & .14) & 14 \\
\hline Major & & & (0) & 0 \\
\hline Total accidents & .68) & $5(26$ & 6.32) & 19 \\
\hline
\end{tabular}

$P=0.00$ I (Chi-square test). ETT: Endotracheal tube

Comparison of accident rates Medical Surgical Obstetric Total by departments from where $\quad(n=105) \quad(n=55) \quad(n=73) \quad(n=233)$ the patients were referred

\begin{tabular}{lcccc}
\hline Number of accidents*** & II & 3 & 5 & 19 \\
Number of patients & 105 & 55 & 73 & 233 \\
Number of TDs & 857 & 303 & 497 & 1657 \\
Airway accidents/patient (\%) & 10.4 & 5.4 & 4.2 & 8.2 \\
Airway accidents/TDs (\%) & 1.2 & 0.9 & 1.0 & 1.1 \\
UE***** & 1 & 0 & 3 & 4 \\
BET***** & 7 & 3 & 0 & 10 \\
BTT***** & 3 & 0 & 2 & 5 \\
\hline
\end{tabular}

*** $P=0.483$ (Chi-square test); **** $P=0.036$ (Chi-square test). TDs: Tube days; UE: Unplanned extubation; BET: Blocked endotracheal tube; BTT: Blocked tracheostomy tube

the tracheostomized group $(P=0.001)$ [Table 1]. There were no major accidents. All except one of the mild accidents were in tracheostomized patients. All except one of the moderate accidents were in the patients with ETT. The proportion of moderate outcomes was higher ETT blockage compared to tracheostomy blockage (90\% vs. $20 \%)(P=0.007)$.

The severity of accidents was affected by the type of accident [Table 2]. The accident rate according to the type of tube (ETT or tracheostomy) was unaffected by the 
Table 2: Severity of airway accidents according to types and influence of shifts, duration of intubation/tracheostomy, and age on accident types

\begin{tabular}{lcccc}
\hline $\begin{array}{l}\text { Comparison of severity of } \\
\text { accident by type of accident }\end{array}$ & $\begin{array}{c}\text { UE } \\
(\boldsymbol{n}=\mathbf{4})\end{array}$ & $\begin{array}{c}\text { BET } \\
(\boldsymbol{n}=\mathbf{1 0})\end{array}$ & $\begin{array}{c}\text { BTT } \\
(\boldsymbol{n}=\mathbf{5})\end{array}$ & $\begin{array}{c}\text { Total } \\
(\boldsymbol{n}=1 \mathbf{9})\end{array}$ \\
\hline Mild & 0 & $\mathrm{I}$ & 4 & 5 \\
Moderate & 4 & 9 & $\mathrm{I}$ & 15 \\
Major & 0 & 0 & 0 & 0 \\
\hline
\end{tabular}

$P=0.006$ (Chi-square test). UE: Unplanned extubation; BET: Blocked endotracheal tube; BTT: Blocked tracheostomy tube

Comparison of accident groups by shifts

\begin{tabular}{lccc}
\hline Shift & ETT group $(n=232)$ & Tracheostomy group $(n=44)$ & Total \\
\hline Day shift & 4 & 0 & 4 \\
Night shift & 10 & 5 & 15 \\
Total & 14 & 5 & 19 \\
\hline
\end{tabular}

$\mathrm{P}=0.179$ (Chi-square test). ETT: Endotracheal tube

Comparison of type of accidents by shifts

\begin{tabular}{lcccc}
\hline Shift & UE $(n=4)$ & BET $(n=10)$ & BTT $(n=5)$ & Total $(n=19)$ \\
\hline Day shift & 4 & 0 & 0 & 4 \\
Night shift & 0 & 10 & 5 & 15 \\
\hline
\end{tabular}

$P=0.00$ I (Chi-square test). UE: Unplanned extubation; BET: Blocked endotracheal tube; BTT: Blocked tracheostomy tube

Comparison of accident types by duration of intubation/tracheostomy

\begin{tabular}{lcccc}
\hline Term & $\begin{array}{c}\text { UE } \\
(n=4)\end{array}$ & $\begin{array}{c}\text { BET } \\
(n=10)\end{array}$ & $\begin{array}{c}\text { BTT } \\
(n=5)\end{array}$ & $\begin{array}{c}\text { Total } \\
(n=19)\end{array}$ \\
\hline Accidents within the first 7 days & 4 & $\mathrm{I}$ & 0 & 5 \\
Accidents after $7^{\text {th }}$ day & 0 & 9 & 5 & 14 \\
\hline
\end{tabular}

$P=0.00$ I (Chi-square test). UE: Unplanned extubation; BET: Blocked endotracheal tube; BTT: Blocked tracheostomy tube

\begin{tabular}{lcccc}
\hline \multicolumn{4}{c}{ Comparison of type of accidents with age } \\
\hline Age (years) & \multicolumn{3}{c}{ Type of accident } & Total \\
\cline { 2 - 4 } & BET & BTT & UE & \\
\hline$<60$ & 5 & 5 & 4 & 14 \\
$\geq 60$ & 5 & 0 & 0 & 5 \\
Total & 10 & 5 & 4 & 19 \\
\hline$P=0.047$ (Chi-square test) UE: Unplanned extubation; BET: Blocked endotracheal tube;
\end{tabular}

$P=0.047$ (Chi-square test) UE: Unplanned extubation; BET: Blocked endotracheal tube; BTT: Blocked tracheostomy tube

shift (day or night) [Table 2]. The type of accidents was affected by the shifts [Table 2]. All UE occurred during day shifts, and all blockages occurred during night shifts.

The type of accident was affected by whether it occurred during the first 7 days of intubation/tracheostomy or after that [Table 2]. Nine of the ten blockages, i.e., $90 \%$ occurred after $7^{\text {th }}$ day of intubation. All UE occurred within the first 7 days of intubation. The severity of the accidents was not affected by the department from where the patients were referred. There was no effect of age group on the incidence of accident (age $\geq 40$ vs. $\angle 40$ [ $P=0.072]$, $\geq 50$ vs. $<50[P=0.234], \geq 60$ vs. $<60[P=0.808])$ and on the severity of accident (age $\geq 40$ vs. $<40[P=0.345]$, $\geq 50$ vs. $<50[P=0.211], \geq 60$ vs. $<60[P=0.709])$. In patients who had accidents, the proportion was higher at night compared to day in the younger age group ( $<40$ years $100 \%$ vs. $\geq 40$ years $50 \%[P=0.008]$ and $<50$ years $100 \%$ vs. $\geq 50$ years $42.85 \%$ [ $P=0.003]$ ). There was association between the type of accidents and age groups. Half of the blockages in ETT patients occurred in the older age group whereas all (100\%) unexplained extubations occurred in younger patients [Table 2].

All ten blockages in the ETT group, three blockages in the TT group, and two UEs occurred in lightly sedated patients (Ramsay sedation scale 2-4 or Richmond agitation-sedation scale -2-0). Two TT blockages and two UEs occurred in awake patients. None of the patients were delirious on the day of airway accidents.

\section{Discussion}

Most of the studies on airway accidents in the ICU focus either on UE of ETT or tracheostomy-related problems. There is only one study analyzing the epidemiological pattern of all airway accidents, which showed that SEs were the most common accidents, majority of which did not require reintubation. ${ }^{[1]}$ The study also showed that tracheostomy accidents were more common than ETT-related ones.

In contrast to previous studies, ${ }^{[1,7,16]}$ blocked tubes were the most common airway accidents in our study and a majority $(93.33 \%)$ occurred after the $7^{\text {th }}$ day of intubation/tracheostomy. This can be attributed to our practice of exclusive usage of HHs and not HMEFs, for humidification. HMEFs could not be afforded due to economic constraints of the patients and lack of free supply by the institution. HMEFs have shown to provide efficient humidification with no occlusion of tracheal tubes for $48 \mathrm{~h}$ to 7 days without changing the filter. ${ }^{[17,18]}$ A study showed that the rate of tracheal tube occlusion, when HMEFs are used, is lower than when HHs are used ${ }^{[19]}$ All of these blockages occurred during night shifts which could be due to the lack of respiratory technician and lower nurse to patient ratio (1:5) during evening and night shifts. All UE (all of them being AEs) in our study occurred during the day shift while carrying out the routine morning care of the patients. Morning care for each intubated or tracheostomized patient was provided by two nurses and one ward assistant, the seniority and experience of whom were highly variable. None of the blockages were major; $33.33 \%$ of them were mild with little or no physiological consequences mostly managed by the resident "on-duty" doctor whereas $66.66 \%$ caused cardiorespiratory decompensation. All blockages and AEs required reintubation.

Recent literature, focused on the problem of UE (SE and $\mathrm{AE}$ ), has suggested that the rate of SE decreased from $8 \%$ to $16 \%{ }^{[4,7-12]}$ during the late 1980 s and early 
1990 s to as much as $3 \%^{[13]}$ in the late 1990 s due to better education of healthcare staff and more vigilant clinical monitoring. ${ }^{[13]}$ More recently, other studies demonstrated that the initiation of a continuous quality improvement program decreased the rate of SE from $2.6 \%$ per TD to $1.5 \%$ and finally to $1.2 \% .{ }^{[20]}$ As compared to the study by Chatterjee et al., ${ }^{[2]}$ the incidence of UE was low in our study $(1.7 \%)$ and 4 per 1077 TDs $(0.4 \%)$. The low UE rate per patient could be due to the fact that a large proportion of patients in our CCU were postoperative with less TDs. There were no SEs. A study with a similar heterogeneous population tended to have a low SE rate. ${ }^{[20]}$ However, less TDs cannot explain the low UE in the term of accidents per TD (4/1077). This can rather be due to the protocol for airway care where even drowsy patients were restrained slightly by tying the hands down so that the hands could be moved a bit but not sufficiently to reach the invasive devices.

In general, it is easier and simpler to maintain TT compared to ETT. The relative ease of percutaneous tracheostomies and apparent decrease of complications associated with this procedure is favoring a trend of early tracheostomies in CCU patients. ${ }^{[21,22]}$ Tracheostomy-related accidents have been recorded to be between $6 \%$ and $66 \% .{ }^{[23-30]}$ The incidence of tracheostomy accidents in this study was $11.4 \%$ per patient and $0.9 \%$ per TD. All tracheostomies were surgical as neither was percutaneous tracheostomy sets affordable for our patients nor was they available free of cost.

Our study showed that the incidence of accidents was similar during endotracheal intubation and tracheostomy; however, unlike previous studies, ${ }^{[1]}$ the incidence of accidents in the ETT group per TD was higher in the tracheostomy group per TD $(1.3 \%$ vs. $0.9 \%)(P=0.001)$, and the outcome of the accidents was more favorable in the patients with tracheostomies compared to ETT. This might be due to the fact that all the tracheostomy-related accidents were blockages, which were attended promptly and managed efficiently by the anesthesia resident on duty.

There were no major accidents. This might be attributed to the fact that our CCU had exclusively anesthesia residents as on-duty doctors, who were competent enough to manage the airway complications promptly. Four out of five $(80 \%)$ of the tracheostomy-related accidents were mild, whereas only $7.1 \%$ of endotracheal-related accidents were mild. The rest were moderate.

Our study was limited by the fact that the study population and accordingly the number of accidents were small, mainly due to the small number of beds in our CCU. The ventilated cardiac surgical and trauma patients are essentially admitted in the exclusive cardiac surgical and trauma ICUs of our institute, and the absence of these groups of patients has also reduced the number of ventilated patients in our general CCU thereby restricting the study population.

\section{Conclusion}

This study showed that the most common accident was blocked tubes, all of which occurred during night shifts when staffing in terms of nurses and respiratory technicians were suboptimal. All UEs occurred in endotracheally intubated patients while patients were moved for the routine morning care. The presence of anesthesiology residents as on-duty doctors in the CCU and availability of senior anesthesiologist' help as and when needed round the clock was probably responsible for successful management of all the airway accidents without any major complication.

\section{Financial support and sponsorship \\ Nil.}

\section{Conflicts of interest}

There are no conflicts of interest.

\section{References}

1. Kapadia FN, Bajan KB, Raje KV. Airway accidents in intubated Intensive Care Unit patients: An epidemiological study. Crit Care Med 2000;28:659-64.

2. Chatterjee A, Islam S, Divatia JV. Airway accidents in an Intensive Care Unit. Indian J Crit Care Med 2004;8:36-9.

3. Amato MB, Barbas CS, Medeiros DM, Magaldi RB, Schettino GP, Lorenzi-Filho G, et al. Effect of a protective-ventilation strategy on mortality in the acute respiratory distress syndrome. N Engl J Med 1998;338:347-54.

4. Coppolo DP, May JJ. Self-extubations. A 12-month experience. Chest 1990;98:165-9.

5. Whelan J, Simpson SQ, Levy H. Unplanned extubation. Predictors of successful termination of mechanical ventilatory support. Chest 1994;105:1808-12.

6. Epstein SK, Nevins ML, Chung J. Effect of unplanned extubation on outcome of mechanical ventilation. Am J Respir Crit Care Med 2000;161:1912-6.

7. Smith RL, Planzos H. Unplanned extubation. Chest 1995;107:887-8.

8. Listello D, Sessler CN. Unplanned extubation. Clinical predictors for reintubation. Chest 1994;105:1496-503.

9. Jayamanne D, Nandipati R, Patel D. Self extubation a prospective study. Chest 1988;94:3S.

10. Seudal I, Garner CV, Kaye W. Accidental extubation in the ICU. Chest 1992;102:1843S.

11. Brown R, Gau P, Touleimat B. Unplanned extubations in a community hospital. Chest 1992;102:183S.

12. O'Neil K. A prospective study of unplanned extubations. Chest 1992;102:183S.

13. Tindol GA Jr., DiBenedetto RJ, Kosciuk L. Unplanned extubations. Chest 1994;105:1804-7.

14. Kapadia FN, Tekawade PC, Nath SS, Pachpute SS, Saverkar SS, 
Bhise RA, et al. A prolonged observational study of tracheal tube displacements: Benchmarking an incidence $<0.5-1 \%$ in a medical-surgical adult Intensive Care Unit. Indian J Crit Care Med 2014;18:273-7.

15. MacIntyre NR, Cook DJ, Ely EW Jr., Epstein SK, Fink JB, Heffner JE, et al. Evidence-based guidelines for weaning and discontinuing ventilatory support: A collective task force facilitated by the American College of Chest Physicians; the American Association for Respiratory Care; and the American College of Critical Care Medicine. Chest 2001;120 6 Suppl: 375S-95S.

16. Ripoll I, Lindholm CE, Carroll R, Grenvik A. Spontaneous dislocation of endotracheal tubes. Anesthesiology 1978;49:50-2.

17. Markowicz P, Ricard JD, Dreyfuss D, Mier L, Brun P, Coste F, et al. Safety, efficacy, and cost-effectiveness of mechanical ventilation with humidifying filters changed every 48 hours: A prospective, randomized study. Crit Care Med 2000;28:665-71.

18. Thomachot L, Leone M, Razzouk K, Antonini F, Vialet R, Martin C. Randomized clinical trial of extended use of a hydrophobic condenser humidifier: 1 vs 7 days. Crit Care Med 2002;30:232-7.

19. Lacherade JC, Auburtin M, Cerf C, Van de Louw A, Soufir L, Rebufat Y, et al. Impact of humidification systems on ventilator-associated pneumonia: A randomized multicenter trial. Am J Respir Crit Care Med 2005;172:1276-82.

20. Kapadia FN. Unplanned extubations. Chest 1995;108:1768-70.
21. Marx WH, Ciaglia P, Graniero KD. Some important details in the technique of percutaneous dilatational tracheostomy via the modified Seldinger technique. Chest 1996;110:762-6.

22. Friedman Y, Fildes J, Mizock B, Samuel J, Patel S, Appavu S, et al. Comparison of percutaneous and surgical tracheostomies. Chest 1996;110:480-5.

23. Stauffer JL, Olson DE, Petty TL. Complications and consequences of endotracheal intubation and tracheotomy. A prospective study of 150 critically ill adult patients. Am J Med 1981;70:65-76.

24. Chew JY, Cantrell RW. Tracheostomy. Complications and their management. Arch Otolaryngol 1972;96:538-45.

25. Skaggs JA, Cogbill CL. Tracheostomy: Management, mortality, complications. Am Surg 1969;35:393-6.

26. Rogers LA. Complications of tracheostomy. South Med J 1969;62:1496-500.

27. Yarington CT Jr., Frazer JP. Complications of tracheostomy. Arch Surg 1965;91:652-5.

28. Dugan DJ, Samson PC. Tracheostomy: Present day indications and technics. Am J Surg 1963;106:290-306.

29. Glas WW, King OJ Jr., Lui A. Complications of tracheostomy. Arch Surg 1962;85:56-63.

30. Meade JW. Tracheotomy - Its complications and their management. A study of 212 cases. N Engl J Med 1961;265:519-23. 